EPJ Web of Conferences 70,00009 (2014)

DOI: $10.1051 /$ epjconf/20147000009

(C) Owned by the authors, published by EDP Sciences, 2014

\title{
The Tsallis Distribution at the LHC
}

\author{
Jean Cleymans ${ }^{1}$ \\ ${ }^{1}$ UCT-CERN Research Centre and Department of Physics \\ University of Cape Town, Rondebosch, South Africa
}

\begin{abstract}
The Tsallis distribution has been used widely in high energy physics to describe the transverse momentum distributions of particles. In this note we show that the use of a thermodynamically consistent form of this distribution leads to a description of identified particles with the same values of the temperature $T$ and the parameter $q$.
\end{abstract}

There exists a rich and wide variety of distributions covering a large range of applications $[1,2]$. Those having a power law behaviour have attracted considerable attention in physics in recent years but there is a long history in other fields such as biology and economics [3].

In high energy physics power law distributions have been applied by a very large number of scientists [4-8] to the description of transverse momenta of secondary particles produced in $p-p$ collisions. Indeed the available range of transverse momenta has expanded considerably with the advent of the Large Hadron Collider (LHC). Collider energies up to $8 \mathrm{TeV}$ are now available in $p-p$ collisions and transverse momenta of hundreds of $\mathrm{GeV}$ are a common occurrence. In this presentation the focus will be on various forms of distributions first proposed by $\mathrm{C}$. Tsallis about twenty-five years ago [9].

In the analysis of the new data, a Tsallis-like distribution gives excellent fits to the transverse momentum distributions as shown by the by the ALICE [6], ATLAS [7] and CMS [8] collaborations at the LHC and by the STAR [4] and PHENIX [5] collaborations at RHIC. In this paper we review the parameterization used by these groups and propose a slightly different one which has a more consistent interpretation and has the bonus of being thermodynamically consistent.

For high energy physics a consistent form of Tsallis statistics (see e.g. [10] and references therein) for the particle number, energy density and pressure is given by the expressions given below

$$
\begin{aligned}
N & =g V \int \frac{d^{3} p}{(2 \pi)^{3}}\left[1+(q-1) \frac{E-\mu}{T}\right]^{-\frac{q}{q-1}} \\
\epsilon & =g \int \frac{d^{3} p}{(2 \pi)^{3}} E\left[1+(q-1) \frac{E-\mu}{T}\right]^{-\frac{q}{q-1}}, \\
P & =g \int \frac{d^{3} p}{(2 \pi)^{3}} \frac{p^{2}}{3 E}\left[1+(q-1) \frac{E-\mu}{T}\right]^{-\frac{q}{q-1}} .
\end{aligned}
$$

where $T$ and $\mu$ are the temperature and the chemical potential, $V$ is the volume and $g$ is the degeneracy factor. As is well-known the Tsallis distribution $[9,11]$ introduces a new parameter $q$ which for transverse momentum spectra is always close to 1, typical values for the parameter $q$ obtained are in the range 1.1 to 1.2 . In the remainder of this paper we will always assume $q>1$.

This is an Open Access article distributed under the terms of the Creative Commons Attribution License 2.0, which permits unrestricted use, distribution, and reproduction in any medium, provided the original work is properly cited. 


\section{EPJ Web of Conferences}

The expressions (1), (2) and (3) are thermodynamically consistent, e.g. it can be easily shown [10] that relations of the type

$$
N=\left.V \frac{\partial P}{\partial \mu}\right|_{T}
$$

are satisfied $[10,12]$. Note that without the extra power of $q$ in the equations (1),(2), (3) the thermodynamic consistency would not be achieved.

It follows from (1) that the momentum distribution is given by,

$$
\frac{d^{3} N}{d^{3} p}=\frac{g V}{(2 \pi)^{3}}\left[1+(q-1) \frac{E-\mu}{T}\right]^{-q /(q-1)}
$$

or, expressed in terms of transverse momentum, $p_{T}$, transverse mass, $m_{T}$, and rapidity $y$

$$
\frac{d^{2} N}{d p_{T} d y}=g V \frac{p_{T} m_{T} \cosh y}{(2 \pi)^{2}}\left[1+(q-1) \frac{m_{T} \cosh y-\mu}{T}\right]^{-q /(q-1)},
$$

At mid-rapidity $y=0$ and for zero chemical potential $\mu=0$ this reduces to

$$
\left.\frac{d^{2} N}{d p_{T} d y}\right|_{y=0}=g V \frac{p_{T} m_{T}}{(2 \pi)^{2}}\left[1+(q-1) \frac{m_{T}}{T}\right]^{-q /(q-1)} .
$$

This is the expression used in $[10,12]$ to fit the LHC transverse momentum spectra.

It is well-known since 1988 [9] that in the limit where the parameter $q$ goes to $1 \mathrm{Eq}$. (6) reduces to the standard Boltzmann distribution:

$$
\lim _{q \rightarrow 1} \frac{d^{2} N}{d p_{T} d y}=g V \frac{p_{T} m_{T} \cosh y}{(2 \pi)^{2}} \exp \left(-\frac{m_{T} \cosh y-\mu}{T}\right) .
$$

The parameterization given in Eq. (7) is close to the one used (but different) e.g. by the ALICE [6], ATLAS [7], CMS [8], STAR [4] and PHENIX [5] collaborations where the following form is used :

$$
\frac{d^{2} N}{d p_{T} d y}=p_{T} \frac{d N}{d y} \frac{(n-1)(n-2)}{n C\left(n C+m_{0}(n-2)\right)}\left[1+\frac{m_{T}-m_{0}}{n C}\right]^{-n}
$$

where $n, C$ and $m_{0}$ are fit parameters. Indeed, after substituting

$$
n \rightarrow \frac{q}{q-1}
$$

and

$$
n C \rightarrow \frac{T+m_{0}(q-1)}{q-1}
$$

The Eq. (9) becomes

$$
\begin{aligned}
\frac{d^{2} N}{d p_{T} d y}= & p_{T} \frac{\mathrm{d} N}{\mathrm{~d} y} \frac{(n-1)(n-2)}{n C\left(n C+m_{0}(n-2)\right)} \\
& {\left[\frac{T}{T+m_{0}(q-1)}\right]^{-q /(q-1)} } \\
& {\left[1+(q-1) \frac{m_{T}}{T}\right]^{-q /(q-1)} . }
\end{aligned}
$$




\begin{tabular}{|l|c|c|}
\hline \multicolumn{3}{|c|}{$p-p$} \\
$900 \mathrm{GeV}$ \\
\hline Particle & $q$ & $T$ \\
\hline$\pi^{+}$ & $1.154 \pm 0.036$ & $0.0682 \pm 0.0026$ \\
$\pi^{-}$ & $1.146 \pm 0.036$ & $0.0704 \pm 0.0027$ \\
$K^{+}$ & $1.158 \pm 0.142$ & $0.0690 \pm 0.0223$ \\
$K^{-}$ & $1.157 \pm 0.139$ & $0.0681 \pm 0.0217$ \\
$K_{S}^{0}$ & $1.134 \pm 0.079$ & $0.0923 \pm 0.0139$ \\
$p$ & $1.107 \pm 0.147$ & $0.0730 \pm 0.0425$ \\
$\bar{p}$ & $1.106 \pm 0.158$ & $0.0764 \pm 0.0464$ \\
$\Lambda$ & $1.114 \pm 0.047$ & $0.0698 \pm 0.0148$ \\
$\Xi^{-}$ & $1.110 \pm 0.218$ & $0.0440 \pm 0.0752$ \\
\hline
\end{tabular}

Table 1. Fitted values of the $T$ and $q$ parameters measured in $p-p$ collisions by the ALICE and CMS collaborations using the Tsallis form (7) for the momentum distribution.

Which, at mid-rapidity $y=0$ and zero chemical potential, has the same dependence on the transverse momentum as (7) apart from an additional factor $m_{T}$ on the right-hand. It has to be pointed out explicitly that the inclusion of the rest mass in the substitution Eq. (11) is not in agreement with the Tsallis distribution as it breaks $m_{T}$ scaling which is present in the Tsallis form (6) but not in Eq. (9). The inclusions of the factor $m_{T}$ leads to a more consistent interpretation of the variables $q$ and $T$ [10, 12].

The distribution (7) has been used to fit the data for identified particles, $\pi, K$ and $p$ for the ALICE [6] collaboration and $K_{s}^{0}, \Lambda$ and $\Xi$ for the CMS [8] collaboration in $p-p$ collisions at $900 \mathrm{GeV}$ [10, 12]. The results are shown in Table 1 for the parameters $T$ and $q$. The coresponding transverse momentum distributions for the ALICE [6] are shown in Fig. (1). For all identified particles the results are consistent with having a system at a Tsallis freeze-out temperature of about

$$
T \approx 70 \mathrm{MeV}
$$

and a value for the $q$ parameter of about

$$
q \approx 1.15
$$

These values are comparable to the ones obtained recently in $[13,14]$ where the original proposal of Hagedorn [15] was extended to a Hagedorn-Tsallis distribution.

The consistency of the values of $q$ is shown in Fig. (2).

In conclusion we can say that the use of the Tsallis parameterization presented in (1) leads to a good description of identified particles in $p-p$ collisions at $900 \mathrm{GeV}$ with a consistent set of parameters. 
EPJ Web of Conferences

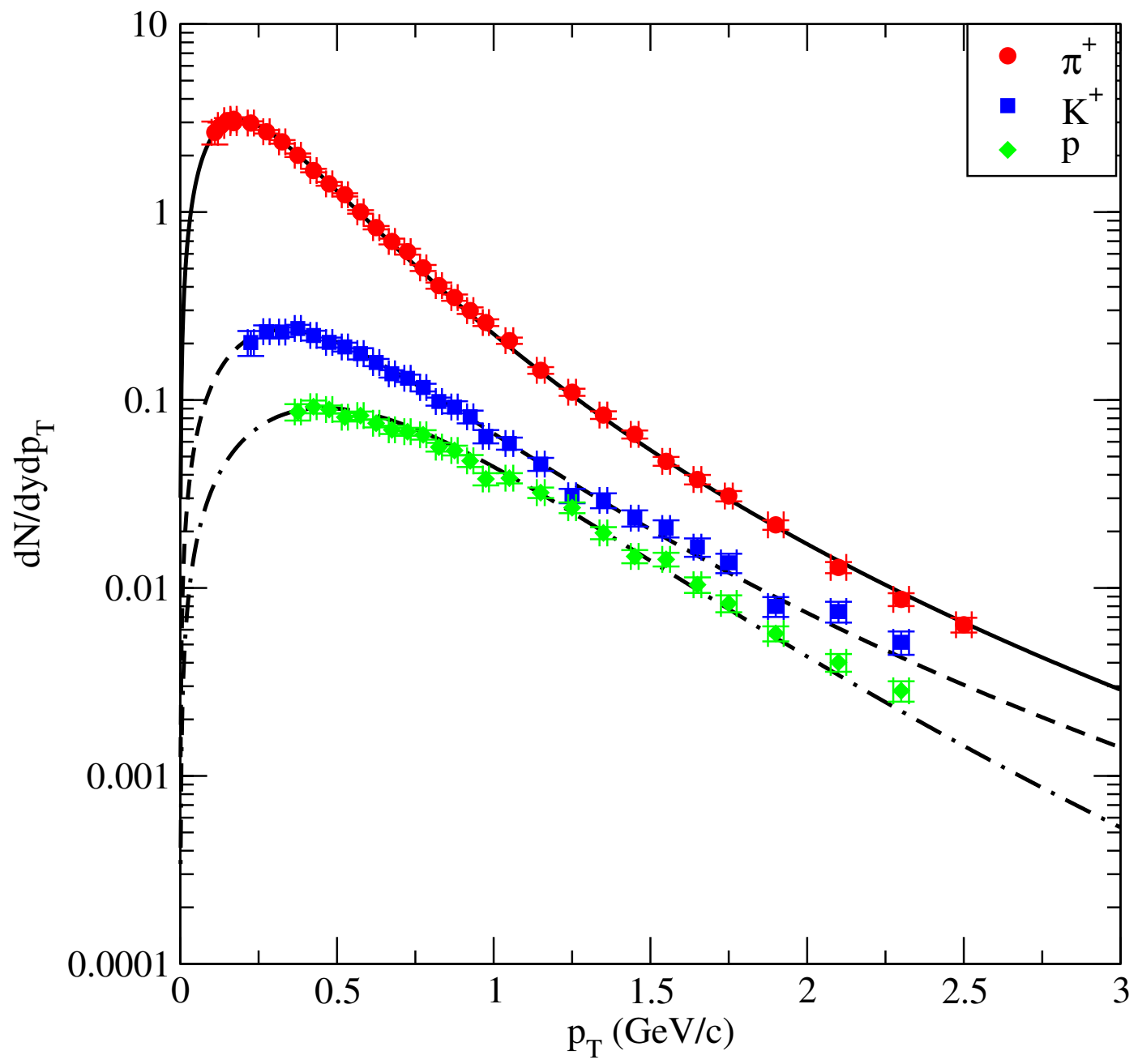

Figure 1. Fit to the $\pi, K, p$ transverse momentum distributions in $p-p$ collisions as measured by the ALICE collaboration [6] using the Tsallis distribution function as given by (7). 
ICFP 2012

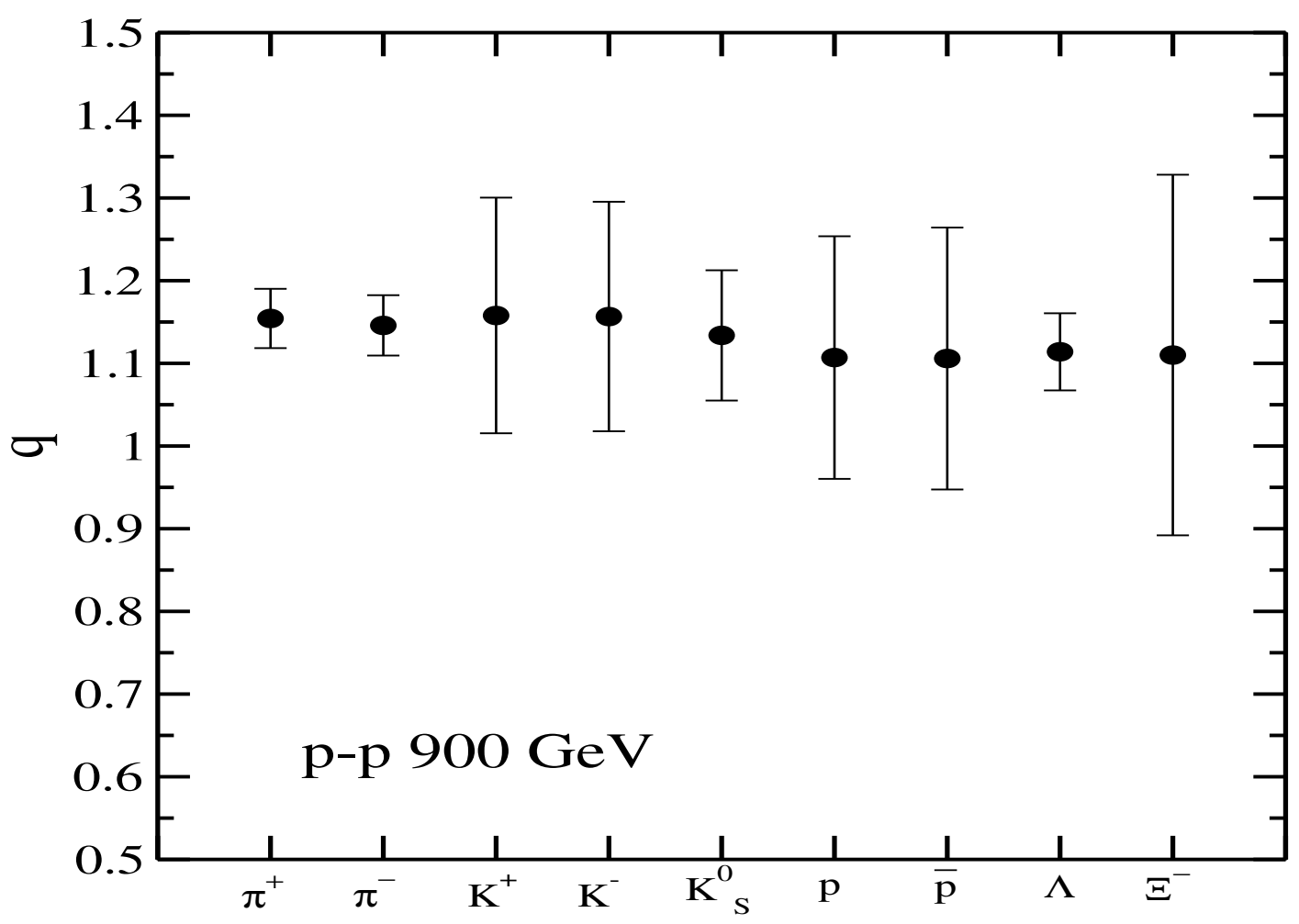

Figure 2. Values of the Tsallis parameter $q$ for different species of hadrons. 


\section{References}

[1] M.E.J. Newman, Contemporary Physics 46, 323 (2005).

[2] A. Clauset, C.R. Shalizi, M.E.J. Newman, SIAM Review 51, 661, 2009.

[3] M. Mitzenmacher, Internet Mathematics, 1, 226 (2003).

[4] B. I. Abelev et al. (STAR Collaboration), Phys. Rev. C 75, 064901 (2007).

[5] A. Adare et al. (PHENIX Collaboration), Phys. Rev. C 83, 052004, (2010); Phys. Rev. C 83, 064903 (2011).

[6] K. Aamodt, et al. (ALICE Collaboration), Eur. Phys. J. C 711655 (2011).

[7] G. Aad, et al. (ATLAS Collaboration), New J. Phys. 13 (2011) 053033.

[8] V. Khachatryan, et al. (CMS Collaboration), JHEP 05, 064 (2011).

[9] C. Tsallis, J.Statist.Phys. 52, 479 (1988).

[10] J. Cleymans and D. Worku, Eur. Phys. J. A (to be published), arXiv:1203.4343.

[11] C. Tsallis, R. S. Mendes, A. R. Plastino, Physica A 261, 534 (1998).

[12] J. Cleymans and D. Worku, J. Phys. G39 025006 (2012) .

[13] A. Deppman, Physica A 391, 6380 (2012).

[14] I. Sena, A. Deppman, arXiv:1208.2952.

[15] R. Hagedorn, Suppl. Nuovo Cimento 3, 147 (2012). 